\title{
ALFVEN SURFACE WAVES ALONG ANNULAR GAS JET SURROUNDING TAR COLUMN UNDER OBLIQUE VARYING MAGNETIC FIELD
}

\author{
AHMED E. RADWAN
}

\begin{abstract}
The MFD oscillating Alfvèn surface waves along a perfectly conducting annular gas jet surrounding a tar column under oblique varying magnetic field has been discussed. Numerous reported works could be recovered as limiting cases. The axial magnetic fields interior and exterior the model are stabilizing while the tenuous azimuthal field is so or not according to restrictions. The thicker the tar column the larger its stabilizing influence; and the oscillation states are slowed down so easier observations are allowed than in the classical case of full fluid jet in vacuum with constant magnetic fields (Uberio and Somasundaram 1980). The Alfvèn wave velocity is decreasing with increasing azimuthal-longitudinal magnetic fields intensities ratio. The phase (dispersive) velocity of the wave propagation relative to Alfvèn wave velocity is monotonic increasing with decreasing the perturbed wavelength.
\end{abstract}

\section{Introduction}

The magnetofluid dynamic (MFD) oscillation of a full fluid jet pervaded by uniform magnetic or electric field is a classical problem. It was Chandrasekhar, Nobel prize winner (1986), who documented such studies and summarized the results of others in his well known book (1961) extending the pioneering works of (3rd) Lord Rayleigh (1945). Uberio and Semasundaram (1980) have studied Alfvèn surface waves along gas column pervaded by uniform magnetic field. Kendall (1986) devised experiments for capillary instability analyses of an annular gas jet by using modern equipments for different amplitude and various wavelengths. The latter author (1986) wrote down about its practical applications in general. Moreover, Kendall (1986) did draw our attention for examining the behaviour of such new fluid systems. In the last decade, Radwan (1988) has elaborated analytically and numerically the capillary instability of a fluid jet coaxial with a tar column endowed with surface tension. We may also refer to the recent work of Radwan (1994) concerning the dynamical behaviour of annular gas-fluid jets.

The purpose of the present work is to investigate the MFD oscillation of Alfvèn surface waves along the interface of annular gas jet, surrounding a tar column upon oblique varying magnetic fields. Such stability studies have crucial applications in the geological domains, for instances the degasification of oil, and other technical applications in engineering plasmas. It may have also a correlation with the applications concerning paint, spraying and crops spraying.

Received June 18, 1996. 


\section{Formulation of the Problem}

We consider a gas cylinder surrounding very dense Tar column. The gas is assumed to be perfectly conducting, inviscid and incompressible. The gas cylinder is of radius a while the radius of the tar column is being $b$ with $b<a$. The model of annular gas jet is pervaded by a homogeneous magnetic field $\left(0,0, H_{0}\right)$ and ambient with the oblique varying magnetic field $\left(0, \beta H_{0} a / r, \alpha H_{0}\right)$ where $\alpha$ and $\beta$ are parameters. We shall use the cylindrical polar coordinates system $(r, \phi, z)$ with the $z$-axis coinciding with the axis of the coaxial gas and tar cylinders. The gas streams with velocity $(0,0, U)$ in the initial state. The model is acting upon the pressure gradient force in addition to the external electromagnetic forces.

The basic magnetofluid dynamics (MFD) equations may be formulated as follows. Interior the annular gas jet:

$$
\begin{aligned}
& \rho \frac{d \underline{u}}{d t}=-\nabla_{p}+\left(\frac{\mu}{2}\right)(\nabla \wedge \underline{H}) \wedge \underline{H} \\
& \frac{\partial \underline{H}}{\partial t}=\nabla \wedge(\underline{u} \wedge \underline{H})-\eta \nabla \wedge(\nabla \wedge \underline{H}) \\
& \nabla \cdot \underline{u}=0 \\
& \nabla \cdot \underline{H}=0
\end{aligned}
$$

In the tenuous medium exterior the annular jet:

Since there is no current

$$
\begin{aligned}
& \nabla \cdot \underline{H}^{\mathrm{ext}}=0 \\
& \nabla \wedge \underline{H}^{\mathrm{ext}}=0
\end{aligned}
$$

Here $p, \underline{u}$ and $\rho$ are the gas kinetic pressure, velocity vector and mass density, $\mu$ is the magnetic permeability coefficient, $\underline{H}$ is the magnetic field intensity and $\eta$ ( $=0$ in our case) is magnetic resistivity.

The MFD equation (eq. (1)) of motion can be written in the form

$$
\frac{\partial \underline{u}}{\partial t}+(\underline{u} \cdot \nabla) \underline{u}-(\mu / 4 \pi \rho)(\underline{H} \cdot \nabla) \underline{H}=-\nabla \Pi
$$

with

$$
\Pi=\rho^{-1}(p+(\mu / 8 \pi)(\underline{H} \cdot \underline{H}))
$$

where $\rho \Pi$ acts as the total MFD pressure which is the sum of the kinetic and magnetic pressures.

For the initial state we have $\underline{u}_{0}=(0,0, U)$ where the subscript 0 will be utilized from now on to indicate unperturbed quantities. For such state, upon using equation (7), we get

$$
\nabla \Pi_{0}=0
$$


This equation implies that the total pressure $\rho \Pi_{0}$ interior the annular gas jet in the initial unperturbed state is constant. The MFD pressure of the annular jet must balance the magnetic pressure in the tenuous medium surrounding the annular jet at the interface $r=a$. This condition gives

$$
p_{0}=\left(\mu H_{0}^{2} / 8 \pi\right)\left(\alpha^{2}+\beta^{2}-1\right)
$$

In view of the physical concept $p_{0} \geq 0$, the parameters $\alpha$ and $\beta$ must satisfy the restrictions

$$
\left(\alpha^{2}+\beta^{2}\right) \geq 1
$$

\section{Perturbation Analysis}

Suppose that the initial state be perturbed, then every perturbed quantity $Q(r, \phi, z ; t)$ can be expressed as

$$
Q(r, \phi, z ; t)=Q_{0}(r)+\delta_{0} Q_{1}(r, \phi, z ; t)
$$

with $Q(r, \phi, z ; t)$ stands for $\underline{u}, \underline{H}, p, \underline{H}^{\text {ext }}$ and the radial distance of the gas cylinder, $\delta_{0}$ is the initial amplitude of the perturbation at $t=0$ (cf. equation (22j). By inserting the expansion (10) into equations (1) - (8), the relevant perturbation equations for the gas jet are given by

$$
\begin{aligned}
& \frac{\partial \underline{u}}{\partial t}+(\underline{u} \cdot \nabla) \underline{u}-(\mu / 4 \pi \rho)\left(\underline{H}_{0} \cdot \nabla\right) \underline{H}_{1}=-\nabla \Pi_{1} \\
& \Pi_{1}=\rho^{-1}\left(p_{1}+(\mu / 4 \pi)\left(\underline{H}_{0} \cdot \underline{H}_{1}\right)\right) \\
& \frac{\partial \underline{H}_{1}}{\partial t}=\nabla \wedge\left(\underline{u}_{1} \wedge \underline{H}_{0}\right)+\nabla \wedge\left(\underline{U}_{0} \wedge \underline{H}_{1}\right) \\
& \nabla \cdot \underline{u}_{1}=0 \\
& \nabla \cdot \underline{H}_{1}=0
\end{aligned}
$$

while those for the tenuous medium surrounding the annular gas jet are given by

$$
\begin{aligned}
& \nabla \cdot \underline{H}_{1}^{\text {ext }}=0 \\
& \nabla \wedge \underline{H}_{1}^{\text {ext }}=0
\end{aligned}
$$

By taking the divergence of equation (10), we get

$$
\left(\frac{\partial}{\partial t}+\left(\underline{u}_{0} \cdot \nabla\right)\right) \nabla \cdot \underline{u}_{1}-(\mu / 4 \pi \rho)\left(\underline{H}_{0} \cdot \nabla\right)\left(\nabla \cdot \underline{H}_{1}\right)=-\nabla^{2} \Pi_{1}
$$

from which, on using equations (14) and (15), we find

$$
\nabla^{2} \Pi_{1}=0
$$

The velocity vector of the gas medium, upon combining equations (11) and (13), may be explicitly obtained. 
For small disturbances, upon considering a single Fourier term and from the linearized theory, the perturebed radial distance of the gas cylinder is

$$
r=a+R_{1}, \quad R_{1} \ll a
$$

with

$$
R_{1}=\delta \cos (k z+m \phi)
$$

Here $m$ and $k$ are the longitudinal and transverse wavenumbers, and $\delta$, the amplitude of the perturbed surface wave, is being

$$
\delta=\delta_{0} \exp \left(-i \sum t\right)
$$

with $\sum$ is the oscillation frequency at time $t$. We have used $\cos (k z+m \phi)$ rather than $\cos k z \cos m \phi$, because it yields a slightly less involved analysis. The arbitrary phase constant has been taken $n \pi / 2$ where $n=1,3,5, \ldots$ by an appropriate choice of the origin of the coordinates.

Since we seek a solution whose $(\phi, z ; t)$-dependence is $\delta \cos (k z+m \phi)$, henceforth the perturbation of any physical quantity $Q_{1}(r, \phi, z ; t)$, based on the linearized theory, can be expressed as

$$
Q_{1}(x, \phi, z ; t)=\delta_{0} Q_{1}(r) \cos (k z+m \phi) \exp \left(-i \sum t\right)
$$

By an appeal to equation (23), the non-singular solution of the system (11)--(19) is given by

$$
\begin{aligned}
\underline{H}_{1} & =\frac{i H_{0}}{\left(\sum+k U\right)} \frac{\partial \underline{u}_{1}}{\partial z} \\
\underline{u}_{1} & =\frac{i\left(\sum+k U\right)}{\left(\Omega_{A}^{2}-\left(\sum+k U\right)^{2}\right)} \nabla \Pi_{1} \\
\Pi_{1} & =\delta\left(\xi I_{m}(k r)+\xi K_{m}(k r)\right) \cos (k z+m \phi)
\end{aligned}
$$

and

$$
\underline{H}_{1}^{\mathrm{ext}}=i C \delta \nabla\left\{K_{m}(k r) \cos (k z+m \phi)\right\}
$$

where in obtaining equation (27), use has been made of equations (16) and (17) and the normal perturbation technique. Here $I_{m}(k r) K_{m}(k r)$ are the modified Bessel functions of the first and second kind of the order $m$, where $\xi, \xi$ and $C$ are arbitrary constants of integration to be determined while

$$
\Omega_{A}=\left(\mu H_{0}^{2} k^{2} / 4 \pi \rho\right)^{\frac{1}{2}}
$$

is the Alfvèn wave frequency defined in terms of $H_{0}$.

\section{Boundary Conditions}

The solution of the basic equations (1)-(8) given by equations (9) and (24)-(28) must satisfy the following boundary conditions. 
[1] The kinematic boundary condition states, for the problem under consideration, that the normal component of the gas velocity must be compatible with the velocity of the boundary surface at $r=a$ and at the same time vanish at $r=b$. These conditions yields

$$
\begin{aligned}
& \xi=-\xi\left(K_{m}^{\prime}(q x) / I_{m}^{\prime}(q x)\right), \quad q=(b / a)<1 \\
& \xi=\left(a^{2} / x\right)\left(\left(\sum+k U\right)^{2}+\Omega_{A}^{2}\right)\left(I_{m}^{\prime}(x) K_{m}^{\prime}(q x)-I_{m}^{\prime}(q x) K_{m}^{\prime}(x)\right)
\end{aligned}
$$

where

$$
x=k a \text { and } q x
$$

are the longitudinal wavenumbers measured with $a$ and $b$ respectively as units of length.

[2] The magnetodynamic condition which states that the normal component of the magnetic field must be continuous at the gas-tenuous medium interface $r=a$. This means that

$$
\underline{N} \cdot<\underline{H}>=0
$$

where $<>$ denotes the jump of $\underline{H}$

$$
<\underline{H}>=\underline{H}^{\mathrm{ex}}-\underline{H}
$$

and $\underline{N}$ is, the unit outward normal vector, given by

$$
\underline{N}=\nabla f /|\nabla f|
$$

where

$$
f(r, \varphi, z ; t)=r-a-R_{1}=0
$$

By the use of equations (20)-(22), (34) and (35); we get

$$
\begin{aligned}
& \underline{N}_{0}=(1,0,0) \\
& \underline{N}_{1}=(0, m, x) \sin (k z+m \varphi)
\end{aligned}
$$

Consequently, the condition (32) yields

$$
C=\frac{-H_{0} a}{x K_{m}^{\prime}(x)}(m \beta+x \alpha)
$$

[3] The magnetofluid dynamic condition: the normal component of the total stress tensor must be continuous across the perturbed gas-tenuous medium interface $r=a$.

This condition leads to the following relation

$$
\left(\sum+k U_{0}\right)^{2}=\frac{\mu H_{0}^{2}}{4 \pi \rho a^{2}}\left\{x^{2}-\left(\beta^{2}+(m \beta+x \alpha)^{2} \frac{K_{m}(x)}{x K_{m}^{\prime}(x)}\right) G_{m}(x, y)\right\}
$$

where

$$
y=q x \text { and } G_{m}(x, y)=\frac{x\left(I_{m}^{\prime}(x) K_{m}^{\prime}(y)-I_{m}^{\prime}(y) K_{m}^{\prime}(x)\right)}{I_{m}(x) K_{m}^{\prime}(y)-I_{m}^{\prime}(y) K_{m}(x)}
$$




\section{Alfvèn Waves}

Following the Uberoi's notations (1980):

$$
\begin{aligned}
& u=1, B=\mu \underline{H} \\
& \varepsilon(k, \omega)=-\rho \omega^{2}+B^{2} k^{2} / 4 \pi, \quad \omega=\sum+k U_{0}
\end{aligned}
$$

he oscillation relation (39) could be rewritten as

$$
\varepsilon(x, \omega)-\frac{B_{0}^{2}}{4 \pi R_{0}^{2}}\left(\beta^{2}+(m \beta+x \alpha)^{2} \frac{K_{m}(x)}{x K_{m}^{\prime}(x)}\right) G_{m}(x, y)=0
$$

Therefore, the phase velocity $V_{s}(=\omega / k)$ is given by

$$
V_{s}^{2}=\frac{\omega^{2}}{k^{2}}=\frac{B_{0}^{2}}{4 \pi \rho}\left\{1-\beta^{2}\left(x^{-2}+\alpha m x \beta^{-1}\right)^{2} \frac{K_{m}(x)}{x^{3} K_{m}^{\prime}(x)} G_{m}(x, y)\right\}
$$

from which

$$
V_{s}=V_{A 1}\left\{1-\gamma^{2}\left(x^{-2}+\alpha m x \beta^{-1}\right)^{2} \frac{K_{m}(x)}{x^{3} K_{m}^{\prime}(x)} G_{m}(x, y)\right\}
$$

where

$$
\gamma=B_{02} / B_{01}
$$

and

$$
V_{A 1}=B_{01} /(4 \pi \rho)^{\frac{1}{2}}
$$

is the Alfvèn wave velocity. Moreover, equation (45) may be written in the form

$$
V_{s}=V_{A 1}\left\{1+\gamma^{2} F_{m}(x, y)\right\}^{\frac{1}{2}}
$$

with

$$
F_{m}(x, y)=-\left(x^{-2}+\alpha m x \beta^{-1}\right)^{2} \frac{K_{m}(x)}{x^{3} K_{m}^{\prime}(x)} G_{m}(x)
$$

It is worthwhile to mention here that equation (48) resembles the result which is given by Uberoi and Somasundaram (1980) (equation 3.2 there) but here with some generalization since we have here annular gas jet pervaded by oblique varying magnetic field. The relation among the phase velocity $V_{s}$ and the Alfvèn wave velocities $V_{A 1}\left(=B_{01} /(4 \pi \rho)^{\frac{1}{2}}\right.$ and $V_{A 2}\left(=B_{01} /(4 \pi \rho)^{\frac{1}{2}}\right.$ may be determined by using equations (46) and (47) as

$$
V_{s}^{2}-V_{A 1}^{2}-V_{A 2}^{2} F_{m}(x, y)=0
$$

From the viewpoint of equation (50) we find that the effect of the curvature on the nature of perturbed Alfvèn surface waves along the gas-tenuous medium of the annular jet appears through the function $F_{m}(x, y)$, independent of the intensities of the magnetic fields interior and exterior the jet. 
Such crucial result has been verified numerically upon $V_{A} / V_{A 1}, G_{m}(x)$ and $F_{m}(x, y)$ as $q \longrightarrow 0$ and $\alpha=0$ in the axisymmetric mode $m=0$, see figure (1). We noticed that the area under the $V_{s} / V_{A 1}$ is decreasing with increasing the values of $\gamma$. This means that the Alfvèn wave velocity is decreasing with increasing the ratio of the tenuous azimuthallongitudinal (interior) magnetic fields. As $B_{02} / B_{01}$, it is found that $V_{A 1} / V_{s}$ is increasing in the range $1.1<x<\infty$ while in the range $0<x<1.1$ it is found there are no waves along the annular gas-tenuous interface. As $x \longrightarrow \infty$ we found that $V_{s} / V_{A 1}$ tends to infinity for all values of $\gamma$, also that $V_{s} / V_{A 1}$ is monotonic increasing with increasing $x$ values.

\section{References}

[1] S. Chandrasekhar, Hydrodynamic and Hydromagnetic Stability, Oxford Univ. Press, Cambr. London. (Dover 1981), 1961.

[2] J. M. Kendall, Phys. Fluids, 29, 2086, 1986.

[3] A. E. Radwan, Indian J. Pure \& Appl. Maths., 19, 1105, 1988.

[4] A. E. Radwan, S. Elazab, and A. Demerdash, Physica Scripta, 50, 142, 1994.

[5] J. W. Rayleigh, The Theory of Sound, Dover Publ., N.Y., 1945.

[6] C. Uberio and K. Somasundaram, Plasma Phys., 22, 747, 1980.

Mathematics Department, Faculty of Science, Ain-Shams University, Cairo, Egypt. 